\title{
The protective action of piperlongumine against mycobacterial pulmonary tuberculosis in its mitigation of inflammation and macrophage infiltration in male BALB/c mice
}

\author{
Nihong Lu, Yongrui Yang, Xiaofei Li, Jie Li, Jie Cheng, \\ Zhengxuan Lv, Yingrong $\mathrm{Du}^{凶}$ \\ Department of Respiratory and Critical Care, The Third People's Hospital of Kunming, \\ Kunming, Yunnan, 650041 China \\ yingrongd7@gmail.com
}

Received: April 2, $2021 \quad$ Accepted: October 26, 2021

\begin{abstract}
Introduction: Piperlongumine (PL) is a bioactive alkaloid and medicinal compound of piperamide isolated from the long pepper (Piper longum Linn). It has demonstrated bactericidal action against Mycobacterium tuberculosis (MTB), the cause of pulmonary tuberculosis; nevertheless, immunomodulatory activity had not been identified for it in MTB-triggered granulomatous inflammation. This study investigated if piperlongumine could inhibit such inflammation. Material and Methods: Mycobacterium tuberculosis strain H37Rv was subjected to a broth microdilution assay. Piperlongumine at 5, 15, and $25 \mu \mathrm{g} / \mathrm{mL}, 0.2 \%$ dimethyl sulphoxide as control or $4 \mu \mathrm{M}$ of dexamethasone were tested in vitro on $\mathrm{MH}-\mathrm{S}$ murine alveolar macrophages. BALB/c mice were orally administered PL at 50, 100 and $150 \mathrm{mg} / \mathrm{kg}$ b.w. after trehalose-6,6-dimycolate (TDM) stimulation. Chemokine and cytokine concentrations were determined in lung supernatants. Flow cytometry and Western blot analysis were performed to determine phosphorylated spleen tyrosine kinase (Syk), c-Jun N-terminal kinase (JNK) and extracellular signal-regulated kinase (ERK) pathways. Results: Piperlongumine inhibited inflammatory mediators and adherence of lymphocyte function-associated antigen 1 to MH-S cells following TDM activation. It also improved macrophage clearance of MTB. In TDM-stimulated MH-S cells, PL significantly influenced the macrophage inducible $\mathrm{Ca}^{2+}$-dependent lectin receptor (Mincle)-Syk-ERK signalling pathway. Oral dosing of PL effectively suppressed the development of pulmonary granulomas and inflammatory reactions in the TDM-elicited mouse granuloma model. Conclusion: PL as an inhibitor of MTB-triggered granulomatous inflammation may be an effective complementary treatment for mycobacterial infection.
\end{abstract}

Keywords: tuberculosis, piperlongumine, macrophages, inflammation, Mincle.

\section{Introduction}

Pulmonary tuberculosis is caused by Mycobacterium tuberculosis, belonging to the Mycobacteriaceae family, which is indicated to be a dangerous pathogen and challenging to treat. In Mycobacterium tuberculosis (MTB) infection, macrophages are the targeted immune cells (1). The host induces quarantine granuloma formation to contain bacteria and prevent their multiplication in response to evasive mycobacterial infection (2). It is possible for an immunocompetent host to overcome minor granuloma-mediated inflammation and thus regulate the infection. In an immunocompromised host; however, granulomas can lead to necrotic granulomatous inflammation which causes widespread tissue destruction (3). A granuloma consists primarily of stimulated macrophages, and $\mathrm{T}$ lymphocytes are scarce in their environment (4). An assemblage of cytokines release caused by Mycobacterium tuberculosis interaction with macrophages can function and modulate the environment of additional cells inside the granuloma (5). Therefore, to modify granuloma-mediated inflammation and control mycobacterial infection, proper control of activated macrophages is necessary.

Many pathogen-associated molecular patterns are associated with MTB pathogenesis through interactions with the pattern recognition receptors. Highly abundant peripheral membrane lipids are key to the virulence of Mycobacterium tuberculosis. Trehalose 6, 6-dimycolate 
(TDM), comprising of peripheral membrane lipids, is the rich lipid formed in cell walls via virulent MTB (6). Infection with this bacterium causes TDM to show immunostimulatory characteristics, including granuloma genesis (7-9). By stimulating the development of cytokines associated with pro-inflammatory mediators such as tumour necrosis factor alpha (TNF- $\alpha$ ), TDM contributes to inflammation and pulmonary granuloma development in mice, reflecting many aspects of normal lung MTB infection (9-13). Thus, the TDM mouse model offers an advantageous means to investigate the responses of granulomas in vivo to various treatments. Release of cytokines and chemokines plays a primary role in the recruitment and aggregation of proinflammatory cells towards the formation of granulomas (10). It has been shown that MTB and its associated components induce the release of inflammatory interleukins such as interleukin 1 beta (IL-1 $\beta$ ), interleukin 6 (IL-6) and TNF- $\alpha$ (11) by mononuclear phagocytes in vitro. Animal tuberculosis models have also shown the presence of these cytokines in granulomas (12). Highly concentrated inflammatory chemokines such as $\mathrm{C}$-C motif chemokine ligand 8 (CXCL-8), C-C motif ligand 2 (CCL-2), C-C motif chemokine ligand 10 (CXCL-10) and C-C motif ligand 5 (CCL-5) have been observed in the BAL bronchial alveolar lavage and serum in tuberculosis patients (13-16). Actions that modify this inflammatory cytokine and chemokine could also improve MTB pathogenesis.

Natural remedies derived from plants provide new medicinal and extremely efficient antimicrobial compounds $(17,18)$. In medical care, one of the leading recent developments in the fight against resistant pathogens involves antimicrobial combination therapy (19-21). Bioactive molecules from plant sources may also be tested for possible effects in the discovery and development of new antibiotics to suppress bacterial resistance (21-23). Bioactive compounds can also potentiate the effect of antimicrobial drugs and plant extracts, thus performing like antibiotic adjuvants (23). The deactivation of a bacterium's mechanism of resistance to existing antibiotics is yet another systematic approach to combating multidrug-resistant microbes in clinical settings. Such plant bioactive substances can also act as inhibitors against infections that are multi-drug-resistant (24-25).

Piperlongumine (PL) is a bioactive alkaloid isolated from the long pepper (Piper longum Linn) used in ancient Indian medicine for the treatment of chronic bronchitis, intestinal cramps and other diseases (26). Piperlongumine, often recognised as piplartin, is a compound of piperamide, which besides being contained by Piper longum $\mathrm{L}$ is also extractable from Indian pepper (27). A bioactive compound (naringenin) is effective against multi-drug-resistant pathogens like Pseudomonas aeruginosa (28). Previous studies have indicated that piperine, an alkaloid present in black pepper, seems to be an effective antimicrobial agent that acts as an efflux pump inhibitor of several bacteria $(29,30)$. Recent piperine and piperlongumine pharmacological research (31-34) has led us to believe that piperamide compounds can strengthen the antibiotic effect of certain traditional antimicrobials significantly, serving as antibiotic adjuvants.

\section{Material and Methods}

Material. Rifampicin and piperlongumine (potency $>98 \%$ ) were obtained from Sigma-Aldrich (Beijing, China).

Culture. Mycobacterium tuberculosis strain H37Rv (ATCC-27294) was cultivated and subsequently inoculated on a laboratory shaker at $37^{\circ} \mathrm{C}$ for 7 days in Middlebrook $7 \mathrm{H} 9$ liquid medium supplemented with $0.4 \%$ glycerol, $0.30 \%$ Tween 80 , and $12 \%$ albumin dextrose catalase to promote organism growth in the exponential phase. The bacterial suspension turbidity was adjusted to $1.0 \mathrm{McF}$ arland standard equivalence $\left(2 \times 10^{7} \mathrm{CFU} / \mathrm{mL}\right)$.

Broth microdilution assay. Piperlongumine was obtained from Sigma-Aldrich. Stock solutions of $1 \mathrm{mg} / \mathrm{mL}$ moxifloxacin hydrochloride (Sigma-Aldrich) were dissolved in water, PL was dissolved in dimethyl sulfoxide (DMSO) at $0.1 \% v / v$, and rifampicin was dissolved in $95 \%$ ethanol. For each compound, the minimum inhibitory concentration (MIC) was determined using the Alamar Blue broth microdilution method (13). In short, $0.04 \%(\mathrm{v} / \mathrm{v})$ of mycobacterial suspensions were prepared between 80 and $0.2 \%$ bovine serum albumin (BSA) so that their turbidities were equivalent to $1.0 \mathrm{McF}$ arland standard. The suspension was diluted in $7 \mathrm{H} 9$ broth at 1:25. The minimum inhibitory concentration was identified as the final drug concentration that caused a bluish to light pink colour change. The MICs calculated for each compound were $0.325 \mu \mathrm{g} / \mathrm{mL}$ for moxifloxacin hydrochloride, $0.5 \mu \mathrm{g} / \mathrm{mL}$ for rifampicin, and $4.5 \mu \mathrm{g} / \mathrm{mL}$ for piperlongumine.

Cell viability assay. An $\mathrm{MH}-\mathrm{S}$ murine alveolar macrophage cell line (MH-S) was obtained from the American Type Culture Collection, (Manassas, VA, USA). Cultured MH-S cells in a $6 \times 10^{5}$ quantity were added to a 96-well plate for $48 \mathrm{~h}$ in the presence or absence of PL $(2.5-45 \mu \mathrm{g} / \mathrm{mL})$ at concentrations 10 times higher than the MIC for the H37Rv strain of M. tuberculosis. The cultured and grown cells were treated with 3-(4,5-dimethylthiazol-2-yl)-2,5-diphenyltetrazolium bromide (MTT) $(1 \mathrm{mg} / \mathrm{mL})$ and then incubated for $3 \mathrm{~h}$ at $37^{\circ} \mathrm{C}$. For total dissolving of formazan crystals, DMSO $(200 \mu \mathrm{L})$ was incorporated into each well and incubated at $37^{\circ} \mathrm{C}$. The plates were read at $587 \mathrm{~nm}$ on a microplate reader with $0.02 \%$ DMSO used as a control. The optical density readings were noted relative to this control.

Cell culture and in vitro treatment. Alveolar MH-S murine alveolar macrophages were grown following the instructions provided by the supplier (Cellular Research Center, Shanghai, China). The TDM was dissolved in chloroform with further dilution with alcohol $(1 \mathrm{mg} / \mathrm{mL})$ and pipetted at $50 \mu \mathrm{g} / \mathrm{well}$ into 96-well plates. The solvent was evaporated for 5-6 h 
at $37^{\circ} \mathrm{C}$ in a chamber. For cell stimulation and incubation, cells were seeded on these plates for 24 or 48 h. Piperlongumine concentrated at $4.5 \mu \mathrm{g} / \mathrm{mL}$, $0.2 \%$ DMSO as the control or $4 \mu \mathrm{M}$ of dexamethasone (Sigma-Aldrich) were added to the wells at the time of cell seeding. Heat-inactivated Bacillus Calmette-Guérin (BCG) activated the cells for $24 \mathrm{~h}$.

RNA isolation and quantitative RT-PCR. The MH-S cells were frozen in RNAiso Plus (Thermo Fisher Scientific, Chengdu, China) after TDM stimulation in the presence or absence of PL. The entire RNA was extracted according to the manufacturer's instructions. Complimentary DNA was synthesised using SuperScript II reverse transcriptase (Thermo Fisher Scientific). A quantitative RT-PCR cDNA analysis was performed to determine the relative expression of mRNA using the $\Delta \Delta \mathrm{Ct}$ method with glyceraldehyde 3-phosphate dehydrogenase as an internal control.

Animals. Male BALB/c mice at 10 weeks old were bought from the Animal Research Centre, Beijing, China. All animal studies were carried out following applicable regulations set out for the maintenance and usage of experimental animals (AKT/231_12/2020-1). In this experiment, mice were injected intravenously with a TDM oil-in-water emulsion. The PL was administered orally to mice at 50,100 , or $150 \mathrm{mg} / \mathrm{kg}$ b.w. for 4 or 7 days. Tween 80 at 5\% was used as a control. Mice were euthanised and samples were examined 4 or 7 days after the TDM challenge.

Pulmonary granulomas were triggered by TDM administration. To intensify the immunomodulatory function, TDM was formulated as an oil-in-water emulsion. The freeze-dried TDM was crushed to $9 \mathrm{~mL}$, then homogenised in $90 \mathrm{~mL}$ of phosphate buffered saline (PBS) vehicle with $2 \mathrm{~mL}$ of Tween 80 . The emulsion comprised $8 \%$ mineral oil, $2 \%$ Tween 80 and 90\% PBS. Mice were injected intravenously (IV) in the tail vein with $100 \mathrm{~mL}$ of emulsion containing $40 \mu \mathrm{g}$ TDM. The mice were administered one of the three PL concentrations 50,100 and $150 \mathrm{mg} / \mathrm{kg}$ b.w. for 3 to 6 successive days from the next day after TDM stimulation. Oral dexamethasone at $0.5 \mathrm{mg} / \mathrm{kg}$ b.w. and $2 \%$ Tween 80 were used as controls. Animals were sacrificed on days 4 and 7 after the TDM stimulation test. The weight of the lungs was measured, and lung weight indices were calculated as stated above (34). For haematoxylin and eosin (H\&E) staining, the lungs were placed in $10 \%$ formalin. The right lobes were maintained using ice-cold saline and homogenised. Supernatants were obtained for the identification of cytokines and chemokines following centrifugation at $8,000 \times g$ for $20 \mathrm{~min}$. The lower right lobes were analysed using flow cytometry.

Determination of concentrations of cytokines and chemokines. Assay kits (ELISA) were used to determine the concentrations of TNF- $\alpha$, IL-10, IL- 6 , and IL-1 $\beta$ cytokines and CCL-2, CCL-5, CXCL-10 and keratinocyte-derived $(\mathrm{KC})$ chemokines.

Flow cytometry. The cells were obtained by trypsinisation with $0.35 \%$ trypsin-EDTA, followed by washing with ice-cold PBS blotting with lymphocyte function-associated antigen 1 (LFA-1) rat anti-mouse monoclonal antibody or IgG1 isotypic antibodies (Invitrogen, Thermo Fisher Scientific, USA) and complexed with goat anti-rat antibody to test the LFA-1 and Mincle expression on murine alveolar macrophages. Cells were examined with a flow cytometer following a quick wash with PBS. The lower lung lobe was used to prepare a single-cell suspension for animal studies by slicing it into tiny pieces and centrifuged at $12,000 \times g$ for $45 \mathrm{~min}$ at room temperature with a solution of $2 \mathrm{mg} / \mathrm{mL}$ Liberase Thermolysin Low (Sigma-Aldrich). The processed lungs were physically distorted by being passed across a sterile $60 \mu \mathrm{m}$ strainer utilising the widest part of the plunger from a $2 \mathrm{~mL}$ syringe. Red blood cells were homogenised with $200 \mathrm{mM} \mathrm{NH}_{4} \mathrm{Cl}, 20 \mathrm{mM}$ $\mathrm{KHCO}_{3}(20 \mathrm{mM})$ and EDTA $(0.2 \mathrm{mM})$. After washing with ice-cold PBS, the cells were isolated and retained in the same buffer, stained with allophycocyanin (APC)labelled lymphocyte antigen 6 complex locus G6D (LY6G) and fluoroisothiocyanate (FITC)-labelled antiCD11b, APC-labelled anti-CD16/32 and phycoerythrin (PE)-labelled anti-CCR3. The samples were evaluated using a cell analyser (BD FACSCalibur, Beijing, China).

Measurement of intracellular phosphorylated signalling molecules by flow cytometry. MH-S cells stimulated by TDM were incubated for $30 \mathrm{~min}$ in a dark area at $37^{\circ} \mathrm{C}$ in a fixation buffer to identify intracellular phosphorylated signalling molecules. After centrifugation for $15 \mathrm{~min}$ at $8,000 \mathrm{rpm}$, a Perm Buffer III (BD Phosflow ${ }^{\mathrm{TM}}$, Beijing, China) permeabilised the cells for $30 \mathrm{~min}$ on ice. Cells were blotted with PE-conjugated anti-mouse or anti-human phospho-p38 mitogen-activated protein kinase (MAPK) (Invitrogen, Thermo Fisher, USA), PE-conjugated anti-mouse or anti-human phosphorus-ERK1/2 (Invitrogen, Thermo Fisher, USA), eFluor660-conjugated anti-mouse or anti-human phosphorus-nuclear factor of kappa light polypeptide gene enhancer in B-cells inhibitor (I $\kappa \beta)$ antibodies or PE/eFluor660-conjugated isotypic antibodies (Invitrogen, Thermo Fisher, USA), followed by washing three times with PBS. Phosphorylated Syk staining was performed using rabbit anti-mouse Syk and FITC-conjugated goat anti-rabbit antibodies. The phosphorylated JNK-staining was performed using anti-mouse p-JNK and APC-conjugated goat anti-mouse antibodies. Cells were exposed for evaluation using a flow cytometric method after brief washing with PBS.

Haematoxylin and eosin (H\&E) staining. The biopsied lungs were set at $4{ }^{\circ} \mathrm{C}$ with $10 \%$ formalin for $72 \mathrm{~h}$, washed with PBS, dried and paraffin fixed. H\&E staining was carried out with ten $5 \mu \mathrm{m}$ aliquots for evaluation of basic architecture and cell infiltration.

Evaluation of PL mitigation of alveolar macrophage intracellular destruction by H37Rv strain of M. tuberculosis. Initially, the H37Rv strain of M. tuberculosis was cultivated at room temperature in Middlebrook $7 \mathrm{H} 9$ medium enriched with $10 \%$ dextrose/catalase/albumin for liquid growth and in Middlebrook $7 \mathrm{H} 10$ medium enriched with $10 \%$ dextrose/catalase/albumin/oleic acid for agar growth. 
Log-phase bacteria were preserved at $-80^{\circ} \mathrm{C}$ as a stock of glycerol $(30 \%)$. Macrophages were infected with M. tuberculosis in an intracellular bacterial survival assay with rifampicin at $1 \mu \mathrm{g} / \mathrm{mL}$ concentration as the control in the absence or presence of PL treatment. After $24 \mathrm{~h}$, the cells were washed and homogenised for $10 \mathrm{~min}$ at room temperature in sterile water and then rinsed using a syringe. The lysed cells were dispersed onto Middlebrook $7 \mathrm{H} 10$ agar plates and incubated to establish the colony-forming units (CFU) at $37^{\circ} \mathrm{C}$.

Determination of Syk and ERK was by Western blot analysis. Radioimmunoprecipitation assay lysis buffer was employed for the collection of cells. To prevent protein degradation, protease and phosphatase inhibitors were added to the lysis buffer. After centrifugation at $20,000 \times g$ for $15 \mathrm{~min}$ at $4^{\circ} \mathrm{C}$, the supernatant was collected and the total proteins were determined with the BCA protein assay. The samples were then placed into each well, separated using $10 \%$ SDS polyacrylamide gels comprising $50 \mu \mathrm{g}$ of protein, and placed onto the PVF membranes. The membrane was blocked in Tris-buffered saline containing $0.3 \%$ Tween 20 (TBS-T) and incubated with primary antibodies diluted with TBS-T with $0.2 \%$ Tween overnight in $5 \% \mathrm{BSA}$ at $4^{\circ} \mathrm{C}$. Anti-phosphorylated Syk, anti-total Syk antibodies, anti-ERK1/2 and anti-phospho-ERK1/2 antibodies were the primary antibodies. The membranes were rinsed with TBS-T and incubated with horseradish peroxidase-conjugated secondary anti-rabbit antibody at room temperature for $2 \mathrm{~h}$, which was diluted with 5\% BSA into the TBS-T. After washing with TBS-T, the enhanced chemiluminescence method was used to visualise immune complexes.

Statistical analysis. Statistical analyses were carried out using GraphPad Prism software, (Graph Pad, San Diego, CA, USA). One-way ANOVA was used to assess the differences between groups, which were deemed to be significant at a $\mathrm{P}$ value of $<0.05$.

\section{Results}

PL suppressed the release of pro-inflammatory chemokines and cytokines from TDM-stimulated lung macrophages. The MTT assay established non-cytotoxic effects in MH-S cells (Fig. 1) and the optimum of the three 5, 15 and $25 \mu \mathrm{g} / \mathrm{mL}$ doses of PL was selected. In all concentrations tested, cell viability following the PL treatment was greater than $97.5 \%$. As shown in Figs 2 a and $2 \mathrm{~b}$, PL treatment greatly decreased the secretion of CCL-2, CCL-5, CXCL-10, TNF- $\alpha$, and IL-6 from TDMstimulated MH-S cells in a dose-dependent manner.

PL weakened mycobacteria's viability in macrophages. The antimicrobial activities of PL have been documented in previous studies $(27,32,34)$, but the impact of PL on the antimicrobial properties of macrophages has not been assessed. The H37Rv intracellularly multiplying strain of $M$. tuberculosis was employed in this study. In this scenario, we treated
M. tuberculosis $\mathrm{H} 37 \mathrm{Rv}$ strain-infected macrophages with PL and evaluated intracellular bacterial endurance after $24 \mathrm{~h}$. As illustrated in Fig. 3, M. tuberculosis survived markedly less well in macrophages when PL at 15 and $25 \mu \mathrm{g} / \mathrm{mL}$ was applied. Meanwhile, substantial impairment of $M$. tuberculosis strain survivability was also demonstrated using rifampicin (1 $\mu \mathrm{g} / \mathrm{mL})$, a frequently employed anti-TB drug. Taken together, these results indicated the suppression by PL of intracellular mycobacterial replication in macrophages.

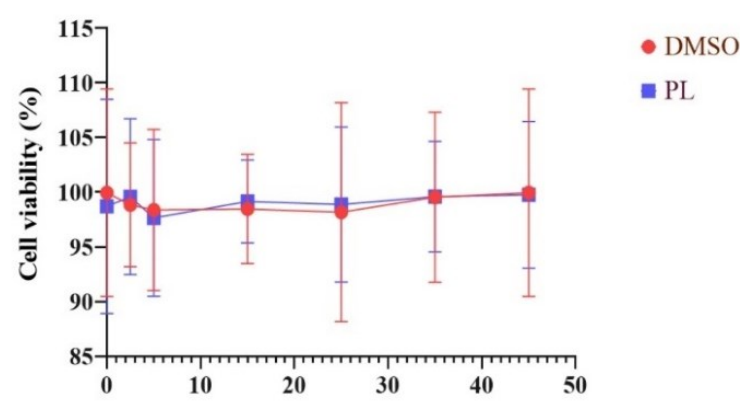

Fig. 1. Effect of piperlongumine (PL) on cell viability in $\mathrm{MH}-\mathrm{S}$ $\left(6 \times 10^{5}\right)$ cells cultured for $48 \mathrm{~h}$ with and without PL $(2.5-45 \mu \mathrm{g} / \mathrm{mL})$. Dimethyl sulphoxide (DMSO) was used as internal reference for comparison. Data are expressed as mean $\pm \mathrm{SD}$ and show no significant differences between DMSO and PL treated cells

In TDM-stimulated MH-S cells, PL influenced the Mincle-Syk-ERK signalling pathway. Thus, we studied if PL affected the TDM-inducing MH-S activation involving intracellular signalling mechanisms. Flow cytometric analyses showed that TDM-triggered surface expression of Mincle was slightly inhibited by PL (Figs $4 \mathrm{a}-\mathrm{c}$ ). In addition, Mincle mRNA expression was substantially suppressed by PL at $25 \mu \mathrm{g} / \mathrm{mL}$. The activity of Syk in TDM-activated MH-S cells was significantly reduced following treatment with PL at 15 and $25 \mu \mathrm{g} / \mathrm{mL}$. MH-S cells display elevated amounts of JNK, ERK and p38 phosphorylation of MAP kinase. Nevertheless, ERK phosphorylation was substantially inhibited by PL treatment at 5,15 and $25 \mu \mathrm{g} / \mathrm{mL}$. Furthermore, no inhibition of I $\kappa \beta$ phosphorylation by PL treatment was observed; however, І $\kappa \beta$ phosphorylation was augmented by the stimulation of TDM. Western blot analysis demonstrated identical inhibitory effects of PL on Syk and ERK protein phosphorylation. Together, the findings showed that in alveolar macrophages, PL inhibited activation of the TDM-induced Mincle-SykERK signalling pathway (Figs $4 \mathrm{a}-\mathrm{c}$ ).

PL diminished granulomatous lung inflammation caused by TDM. The formation of granulomas in mouse lungs has been documented to be attributed to IV administration of emulsified TDM to mice. As seen in Fig. 5, after the TDM challenge, tiny central clusters produced by cell aggregates were recorded from day 4 . On day 7, the clusters were more dynamic. There were no major signs of inflammation or granuloma development with 5\% Tween 80 after stimulation with TDM. The inflammation and granuloma caused by TDM were prevented by PL administration in a dose and timedependent manner (Fig. 5). Following treatment with 50 and $100 \mathrm{mg} / \mathrm{kg}$ b.w. doses of PL for 7 days, virtually 
no aggregation or granulomas were detected. The oral dosing of dexamethasone at $0.5 \mathrm{mg} / \mathrm{kg}$ greatly increased TDM-induced lung consolidation. As determined by the lung weight indices, TDM-induced inflammatory swelling of the lungs decreased markedly with PL at doses of 100 and $150 \mathrm{mg} / \mathrm{kg}$ b.w. for 4 and 7 days.

In mice, the TDM-triggered release of pro-inflammatory chemokines and cytokines was suppressed by PL. Our in vitro analysis made it evident that PL inhibited the cytokines and pro-inflammatory cytokines. TDM activated mouse alveolar macrophage chemokines. The level of expression of TNF-alpha, IL-1 $\beta,-6, \mathrm{KC}, \mathrm{CCL}-2$, CCL-5, and CXCL-10 was further investigated in TDMtreated murine lung tissue. As shown in Fig. 6, when no other substances were administered, TDM greatly potentiated the secretion of these pro-inflammatory cytokines and chemokines after 4 and 7 days of application. Many of them were expressed to a fourfold greater extent in response to the TDM challenge. Nevertheless, with the effect of PL, all cytokines examined were inhibited without major variations on day 4 (Fig. 6). The chemokine amounts were also reduced, but only $\mathrm{KC}$ demonstrated a substantial decrease after treatment with PL at 100 and $150 \mathrm{mg} / \mathrm{kg}$ b.w. Conversely, from day 7 , all cytokines measured excluding TNF- $\alpha$ showed a substantial decline at all three PL concentrations tested. Chemokine suppression was also found in TDM-stimulated PL-treated mice; PL treatment at $100 \mathrm{mg} / \mathrm{kg} \mathrm{b.w}$. displayed the most significant inhibitory outcomes in KC, CCL-2, and CCL-5 while the $50 \mathrm{mg} / \mathrm{kg}$ b.w. concentration was the most significant suppressor of CXCL-10. The level of IL-10 expression in the murine lungs after 6 days of treatment with PL was also evaluated. Figure 6 indicates that there was no apparent rise in IL-10 expression after the TDM challenge, and that PL also had no apparent impact on it. The suppressive effect of PL on proinflammatory chemokines and cytokines expression was demonstrated by these findings.

PL suppressed infiltration of macrophages and neutrophils in pulmonary TDM-treated mice. Various leukocytes were enumerated in the lungs to assess the impact of PL on cell infiltration in pulmonary granulomas. Flow cytometric determinations were performed on day 7 after the TDM challenge. As illustrated in Fig. 7, after 7 days of the TDM test, the numbers of macrophages and neutrophils were substantially elevated. Upon TDM stimulation, macrophage and neutrophil numbers increased 3.07- and 2.13-fold, respectively. However, the aggregation of macrophages in the lungs of TDM-challenged mice was inhibited in all cases without substantial variations following oral administration of PL at different concentrations. Furthermore, PL at 200 and $300 \mathrm{mg} / \mathrm{kg}$ b.w. greatly decreased the number of infiltrating mouse lung neutrophils. A previous study showed that by triggering macrophages, eosinophils can worsen the course of mycobacterial infection (43). We found that after the TDM challenge, the number of eosinophils in the lungs increased significantly, and no apparent inhibition of eosinophils was noticed at any tested concentration of PL (Fig. 7). In line with a previous study (44), macrophages were the most prevalent form of leukocyte in TDM-stimulated murine lung tissue and were approximately 3 and 10 times more abundant than neutrophils and eosinophils, respectively (Fig. 7). These observations collectively indicate that the administration of PL inhibited the recruitment of leukocytes and particularly macrophages and neutrophils into mouse lung granulomas induced by TDM.
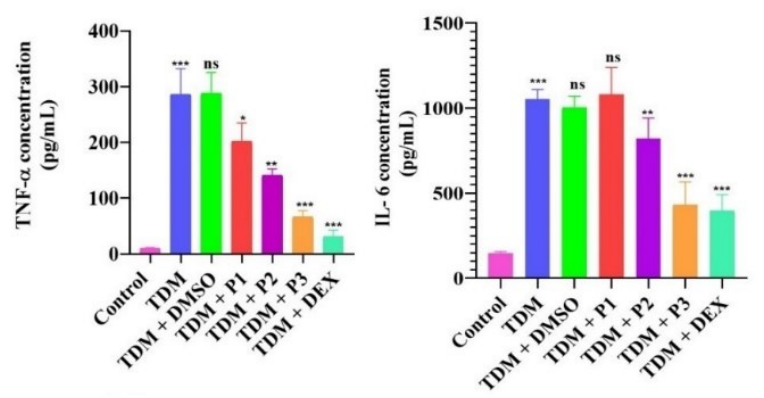

Fig. 2a. In vitro effects of piperlongumine (PL) on the inhibition production of TNF- $\alpha$ and IL-6 in trehalose 6,6-dimycolate (TDM)-activated MH-S cells. Data are expressed as mean $\pm \mathrm{SD}$ of three experiments. DMSO - dimethyl sulphoxide; P1 - PL at $5 \mu \mathrm{g} / \mathrm{mL}$; P2 - PL at $15 \mu \mathrm{g} / \mathrm{mL}$; $\mathrm{P} 3$ - PL at $25 \mu \mathrm{g} / \mathrm{mL}$; DEX - dexamethasone; ns - non-significant, $\mathrm{P}>0.05 ; * \mathrm{P}<0.05 ; * * \mathrm{P}<0.01 ; * * * \mathrm{P}<0.001$ vs TDM-treated group
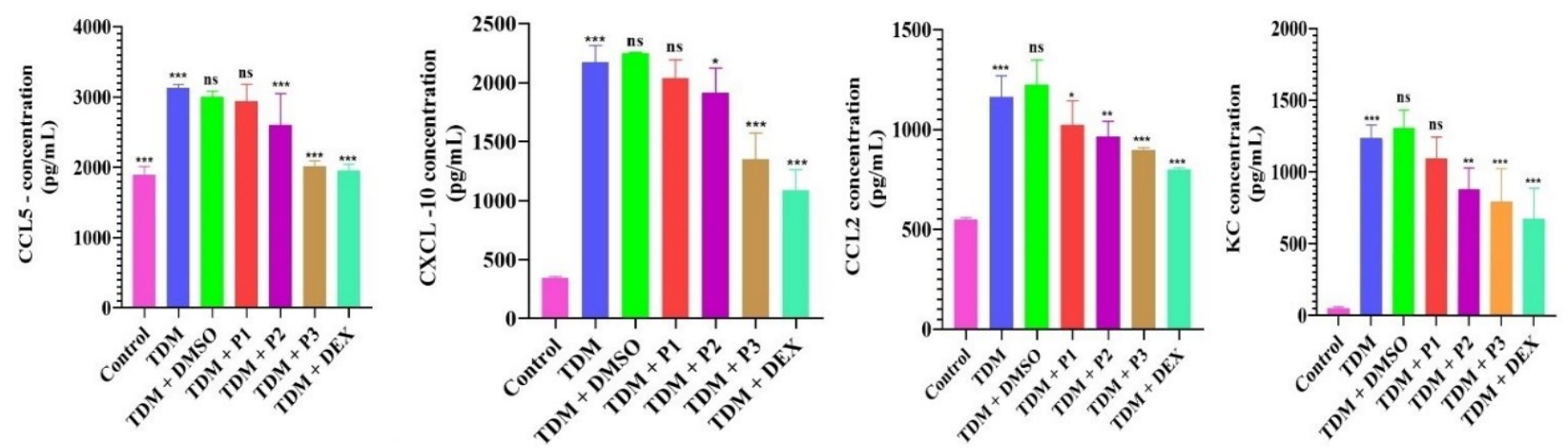

Fig. 2b. In vitro effects of piperlongumine (PL) on the inhibition of production of C-C motif chemokine ligand 2 (CCL-2), C-C motif chemokine ligand 10 (CXCL-10), C-C motif chemokine ligand 5 (CCL-5) and keratinocyte-derived chemokine (KC) in trehalose 6,6-dimycolate (TDM)activated MH-S cells. Data are expressed as mean \pm SD of three experiments. DMSO - dimethyl sulphoxide; P1 - PL $5 \mu \mathrm{g} / \mathrm{mL} ; \mathrm{P} 2-\mathrm{PL} 15 \mu \mathrm{g} / \mathrm{mL}$; P3 - PL $25 \mu \mathrm{g} / \mathrm{mL}$; DEX - dexamethasone; ns - non-significant, $\mathrm{P}>0.05 ; * \mathrm{P}<0.05 ; * * \mathrm{P}<0.01 ; * * * \mathrm{P}<0.001$ vs TDM-treated group 


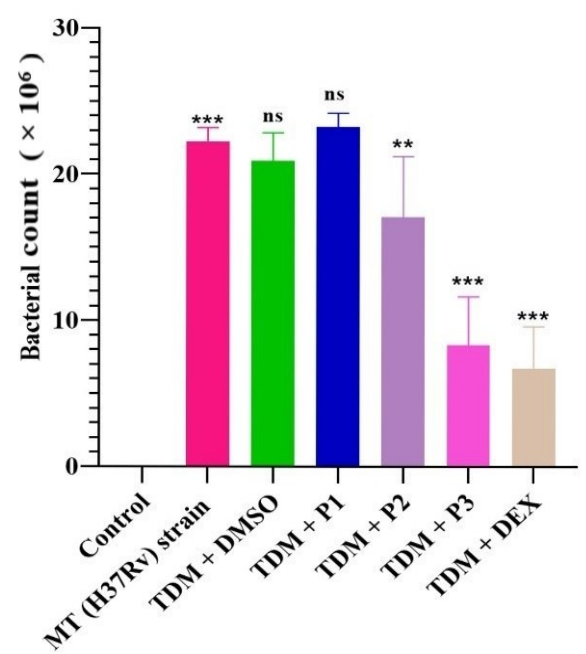

Fig. 3. Enhancing effect of piperlongumine (PL) on the clearance of mycobacterium from alveolar macrophages. Data are expressed as mean $\pm \mathrm{SD}$ of three experiments. TDM trehalose-6,6-dimycolate; DMSO - dimethyl sulphoxide; P1 - PL at $5 \mu \mathrm{g} / \mathrm{mL} ; \mathrm{P} 2$ - PL at $15 \mu \mathrm{g} / \mathrm{mL} ; \mathrm{P} 3-\mathrm{PL}$ at $25 \mu \mathrm{g} / \mathrm{mL} ; \mathrm{DEX}$ - dexamethasone; ns - non-significant, $\mathrm{P}>0.05$; ** $\mathrm{P}<0.01 ; * * * \mathrm{P}<0.001$ vs M. tuberculosis H37Rv strain infection group
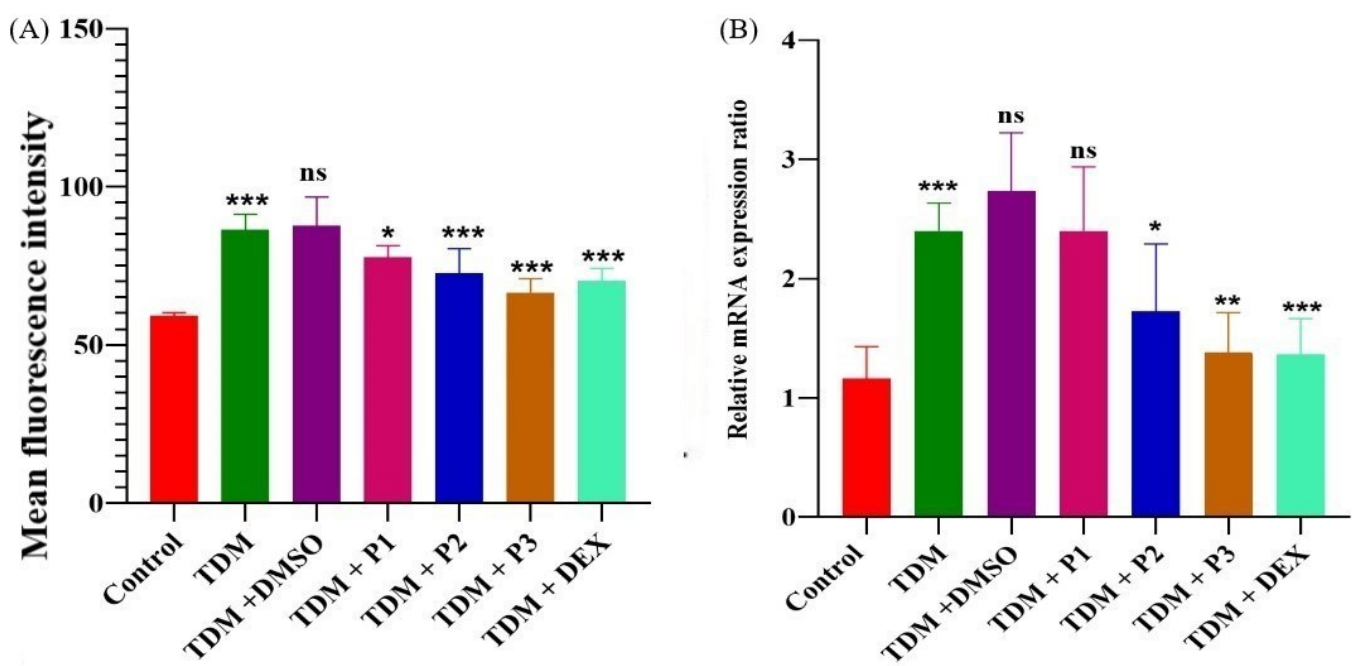

Fig. 4a. The effect of piperlongumine (PL) on the Mincle-Syk-ERK signalling pathway in trehalose 6,6-dimycolate (TDM)-activated MH-S cells. A) Quantification of surface Mincle expression on MH-S cells. B) Mincle mRNA expression from MH-S cells analysed using RT-PCR. Values are represented as a fold of shift relative to non-stimulated and non-treated controls. Data are expressed as mean $\pm \mathrm{SD}$ of three experiments. DMSO dimethyl sulphoxide; P1 - PL at $50 \mathrm{mg} / \mathrm{mL}$; P2 - PL at $100 \mathrm{mg} / \mathrm{mL}$; P3 - PL at $150 \mathrm{mg} / \mathrm{mL}$; DEX - dexamethasone; ns - non-significant, P > 0.05; $* \mathrm{P}<0.05 ; * * \mathrm{P}<0.01 ; * * * \mathrm{P}<0.001$ vs TDM-treated group
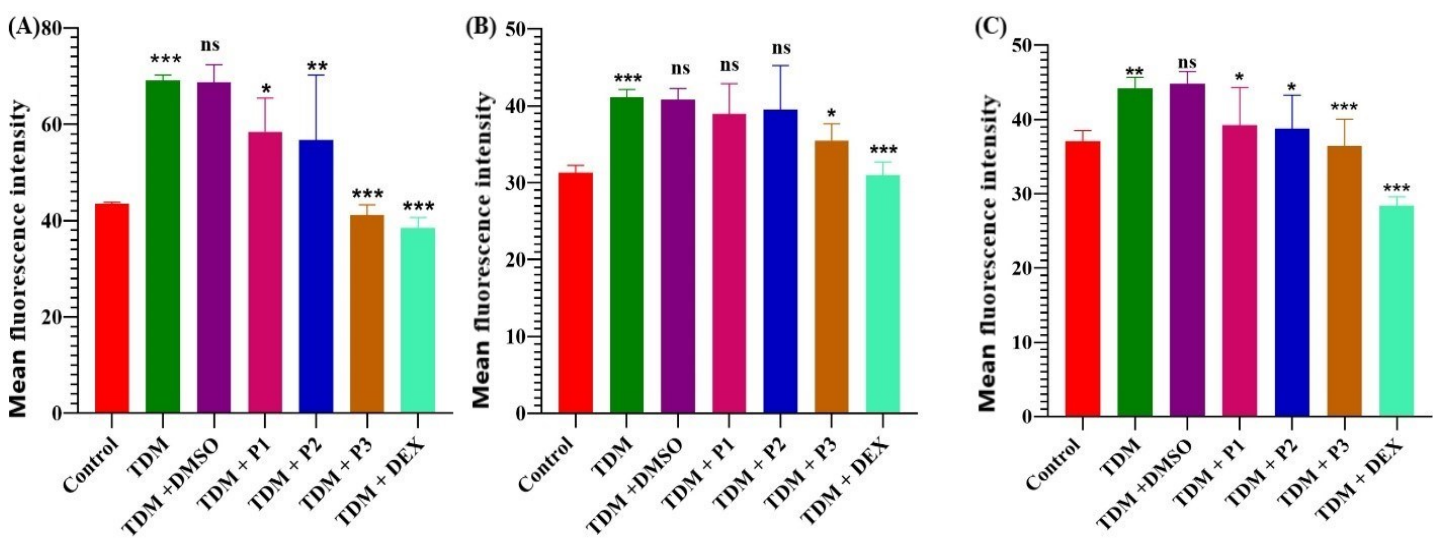

Fig. 4b. The effect of piperlongumine (PL) on the Mincle-Syk-ERK signalling pathway in trehalose 6,6-dimycolate (TDM)-activated MH-S cells. Data are expressed as mean \pm SD of three experiments. A) Intracellular levels of phosphorylated Syk. B) Phosphorylated JNK. C) Phosphorylated ERK. P1 - PL at $50 \mathrm{mg} / \mathrm{mL} ; \mathrm{P} 2$ - PL at $100 \mathrm{mg} / \mathrm{mL} ; \mathrm{P} 3$ - PL at $150 \mathrm{mg} / \mathrm{mL}$; DEX - dexamethasone; ns - non-significant, P $>0.05 ; *$ P $<0.05 ; * *$ $\mathrm{P}<0.01 ; * * * \mathrm{P}<0.001$ vs TDM-treated group 

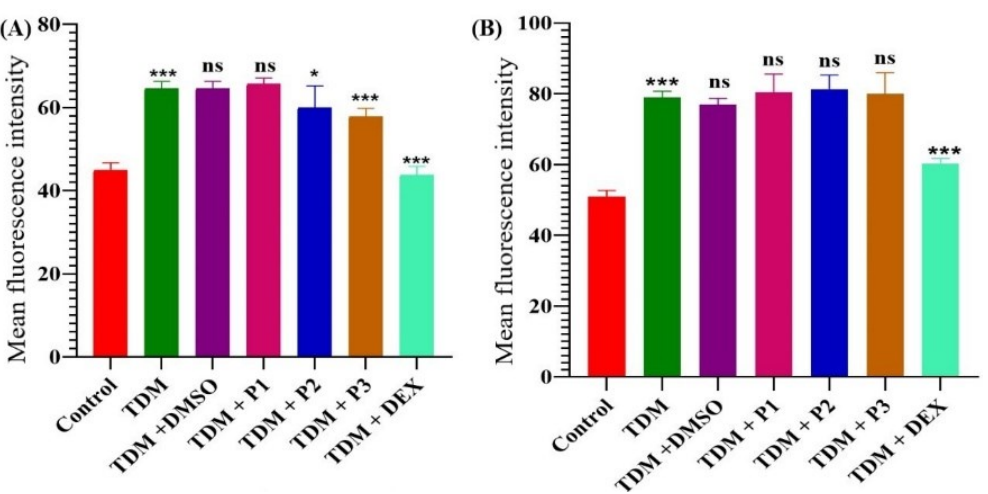

Fig. 4c. The effect of piperlongumine (PL) on the Mincle-Syk-ERK signalling pathway in trehalose 6,6-dimycolate (TDM)-activated MH-S cells. Data are expressed as mean \pm SD of three experiments. A) Phosphorylated p38 mitogen-activated protein kinase (MAPK). B) Phosphorylated nuclear factor of kappa light polypeptide gene enhancer in B-cells inhibitor (Iк $\beta)$. P1 - PL at $50 \mathrm{mg} / \mathrm{mL}$; P2 - PL at $100 \mathrm{mg} / \mathrm{mL}$; P3 - PL at $150 \mathrm{mg} / \mathrm{mL}$; DEX - dexamethasone; ns - non-significant, $\mathrm{P}>0.05 ; * \mathrm{P}<0.05 ; * * \mathrm{P}<0.01 ; * * * \mathrm{P}<0.001$ vs TDM treated group

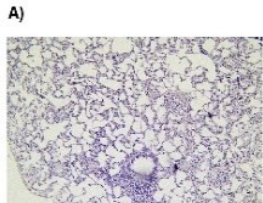

A) Control

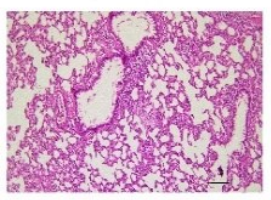

D) PL (100 mg/kg)

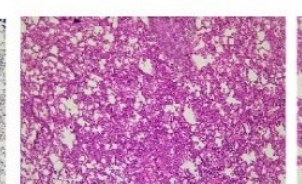

B) TDM

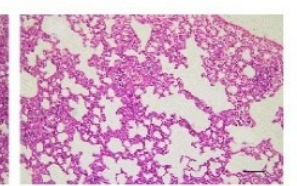

C) PL (50 mg/kg)

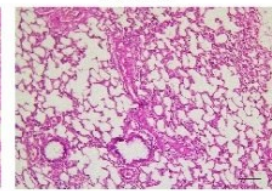

E) PL (150 mg/kg)
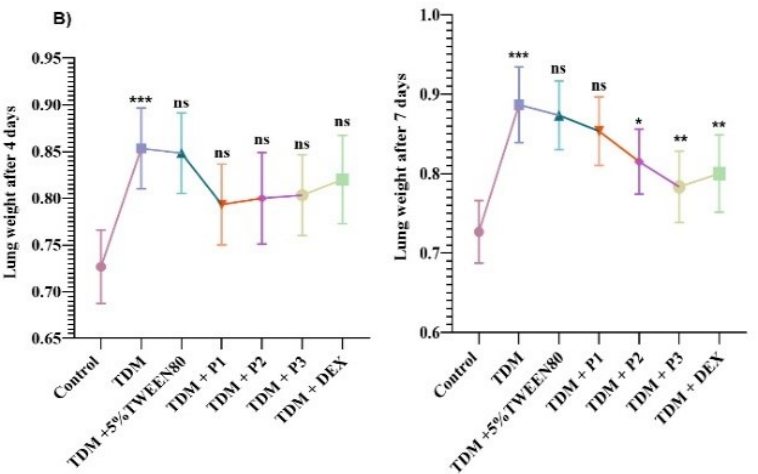

Fig. 5. Diminution by piperlongumine of trehalose 6,6-dimycolate (TDM)-stimulated pulmonary granulomatous inflammation in BALB/c mice. Data are expressed as mean $\pm \mathrm{SD}$ of three experiments. A) Haematoxylin and eosin-stained granulomatous response in BALB/c mice after 4-7 days of piperlongumine (PL) treatment after TDM challenge. B) Inflammatory intensity level in lungs removed from TDM-challenged mice on days 4 and 7. P1 - PL at $50 \mathrm{mg} / \mathrm{mL}$; P2 - PL at $100 \mathrm{mg} / \mathrm{mL}$; P3 - PL at $150 \mathrm{mg} / \mathrm{mL}$; DEX - dexamethasone; ns - non-significant, P > 0.05; $* \mathrm{P}<0.05 ; * * \mathrm{P}<0.01 ; * * * \mathrm{P}<0.001$ vs TDM-treated group
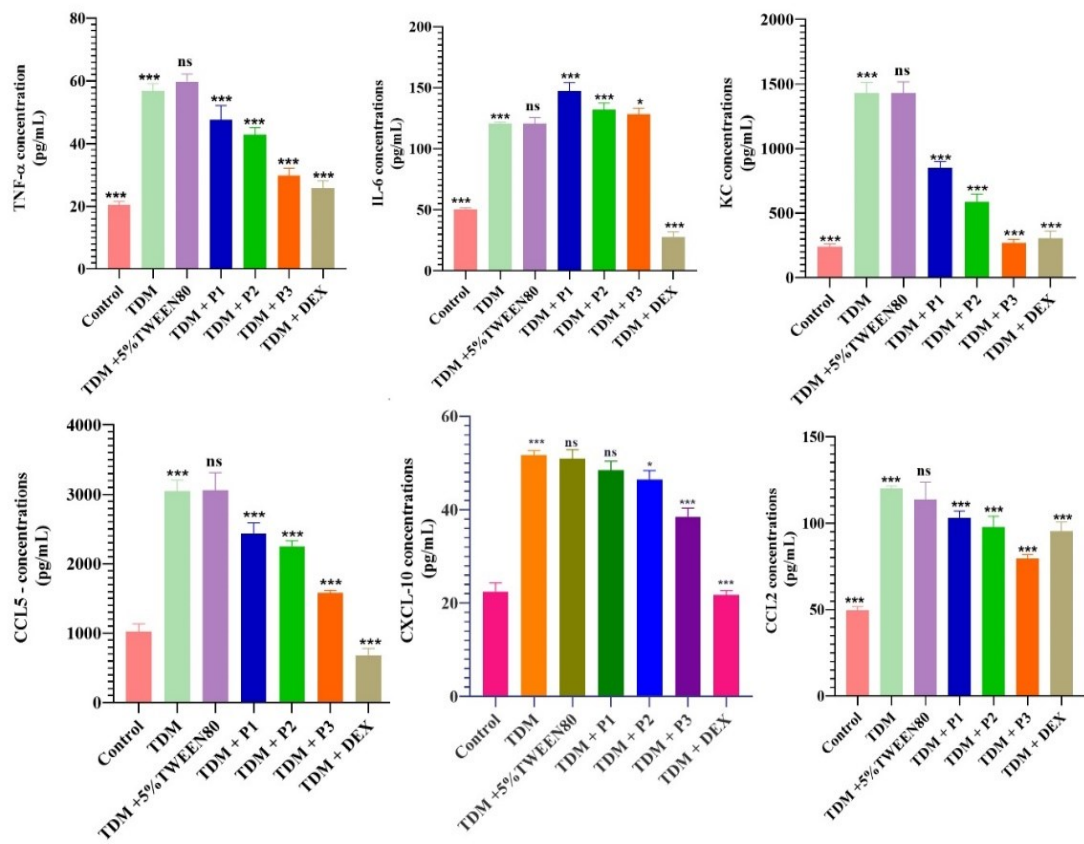

Fig. 6. Effect of piperlongumine on trehalose 6,6-dimycolate (TDM)-stimulated pulmonary granulomatous inflammation in BALB/c mice. Data are expressed as mean \pm SD of three experiments. Graphs show production of tumour necrosis factor alpha (TNF- $\alpha$ ), interleukin 6 (IL-6), interleukin 10 (IL-10), keratinocyte-derived cytokine (KC), C-C motif chemokine ligand 5 (CCL-5), C-C motif chemokine ligand 10 (CXCL-10) and C-C motif chemokine ligand 2 (CCL-2) in pulmonary homogenates after 4 and 7 days of TDM stimulation with or without piperlongumine (PL) treatment. P1 - PL at $50 \mathrm{mg} / \mathrm{mL} ; \mathrm{P} 2-\mathrm{PL}$ at $100 \mathrm{mg} / \mathrm{mL} ; \mathrm{P} 3-\mathrm{PL}$ at $150 \mathrm{mg} / \mathrm{mL} ; \mathrm{DEX}-$ dexamethasone; ns - non-significant, $\mathrm{P}>0.05 ; * \mathrm{P}<0.05 ; * * \mathrm{P}<0.01$; $* * * \mathrm{P}<0.001$ vs TDM-treated group 

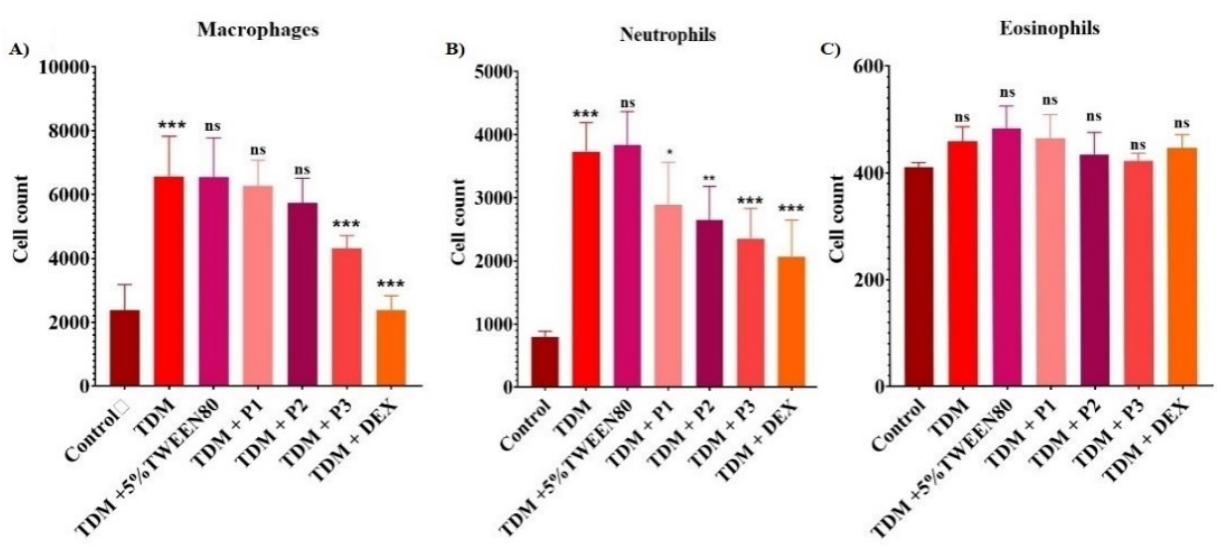

Fig. 7. Inhibition by piperlongumine (PL) of leukocyte infiltration in trehalose 6,6-dimycolate (TDM)-stimulated pulmonary granulomatous inflammation in BALB/c mice. Data are expressed as mean \pm SD of three experiments. A) CD11b+ Ly6G-macrophages. B) CD11b + lymphocyte antigen 6 complex locus G6D (Ly6G+)-neutrophils. C) chemokine receptor type 3 (CCR3)+ CD16/32-eosinophils. Bar charts represent quantitative measurement of positively stained cells. P1 - PL at $50 \mathrm{mg} / \mathrm{mL}$; P2 - PL at $100 \mathrm{mg} / \mathrm{mL}$; P3 - PL at $150 \mathrm{mg} / \mathrm{mL}$; DEX - dexamethasone; ns - non-significant, $\mathrm{P}>0.05 ; * \mathrm{P}<0.05 ; * * \mathrm{P}<0.01 ; * * * \mathrm{P}<0.001$ vs TDM-treated group

\section{Discussion}

Inflammation and continued production of granulomas is the basis of an effective and adequate host immune response to evasive mycobacterial infection (43). Extreme granuloma-triggered inflammation, however, is still the major source of damaging pulmonary injury (2). A promising solution to the treatment of TB is known to address the production of inflammatory mediators. A variety of clinical studies utilising anti-inflammatory medication in combination with chemotherapeutic drugs found this modality to have accelerated MTB clearance and improved therapeutic effects (38). Herbal medicines have also been widely supportive of the immunomodulatory function in the additional treatment of TB. Previous research has shown that PL is anti-inflammatory and antibacterial. Nonetheless, PL's anti-inflammatory and antibacterial effects and their mechanisms, particularly in tuberculous granulomas have not been investigated. In the present research, we illustrated the immune modulatory function of PL in inflammation induced by TDM to mimic the granulomatous inflammation arising in TB. Piperlongumine effectively inhibited the activation of murine alveolar macrophages responding to TDM by suppressing the Mincle-Syk-ERK signalling pathway.

Furthermore, treatment with PL decreased mouse lung inflammation and inflammatory cell invasion, thus alleviating TDM-induced lung injuries. The function of alveolar macrophages against tuberculosis and in the production of granulomas is crucial. Pattern recognition receptors for MTB are sensed by the macrophages, and their detection triggers enhanced cytokine output, inflammatory response, and consequently granuloma development. Our findings demonstrated that TDM mediated the synthesis and production of inflammatory chemokines and cytokines via macrophages, in agreement with an earlier report (39). Without inducing cytotoxicity, PL substantially inhibited the production of cytokines and chemokines (Figs 1 and 2). Lung epithelial cells that are active in the removal of pathogens are often important for the preservation of the mucous membranes because they modify the function of pulmonary macrophages. The current study demonstrated that PL suppressed TDM-stimulated macrophages through the pro-inflammatory chemokine and cytokine release (Fig. 2).

However, the production of pro-inflammatory mediators can also be suppressed by dexamethasone. Different pathways may be exploited by PL to those of dexamethasone. Some diffusible mediators produced by lung alveolae may be influenced by PL. In addition to its effectiveness in suppressing chemokines and cytokines in pulmonary macrophages, PL's action when administered at 15 and $25 \mu \mathrm{g} / \mathrm{mL}$ greatly inhibited the development of CCL-2 macrophage chemokines. Furthermore, it substantially reduced the LFA-1 expression on the surfaces of TDM-activated MH-S cells (44).

These results indicate that PL will effectively inhibit TDM-mediated alveolar macrophages, possibly by influencing the secretion and expression of CCL-2 in $\mathrm{MH}-\mathrm{S}$ cells through its effect on LFA-1. Heatinactivated BCG was used for the activation of pulmonary macrophages to additionally examine the impact of PL in vitro on TB-mediated inflammation. In BCG-activated macrophages, PL administration inhibited the pro-inflammatory cytokine and chemokine expression. Dexamethasone, however, effectively reduced chemokine and cytokine release in macrophages regardless of culture processes. PL also inhibited LFA-1 expression and CCL-2 release in BCG-enabled macrophages, leading to the suppression of macrophage migration. An intracellularly-growing M. tuberculosis strain H37Rv was used to determine whether PL impaired the antimicrobial activities of macrophages, but PL in fact potentiated the destruction of bacteria in macrophages. These findings demonstrate the in vitro anti-intracellular and anti-inflammatory effects of PL against mycobacteria.

It was reported in earlier studies that the C-type lectin receptor Mincle is the dynamic receptor for the action of $\operatorname{TDM}(35,36)$. Mincle is expressed in macrophages and is therefore not controlled by TDM stimulation (37). Through its involvement, Syk signalling plays a key role in the stimulation of innate immune cells induced by TDM (38). Syk phosphorylation subsequently activates the signalling of 
MAP, which has pro-inflammatory outcomes $(39,40)$. Syk, in the pathway with Mincle and along with those of NF-k $\beta$ and MAPK, was suggested to have a significant role in granulomatous inflammation as an intracellular signalling mechanism associated with TDM-mediated transduction of adhesion molecules and escalation of pro-inflammatory chemokine and cytokine secretion (45, 46). The NF-k $\beta$ protein complex has also been noted as significant in triggering TDM-mediated inflammation in other studies (41). Our findings demonstrated that Mincle expression was intensified by TDM stimulation, thus indicating that its surface expression and the amount of mRNA were inhibited by treatment with PL. Following TDM stimulation, the Syk and MAPK kinases JNK, ERK and p38 were the upregulated downstream Mincle molecules. However, the phosphorylation of Syk and ERK was only greatly suppressed by PL and was not by dexamethasone. This is the first research demonstrating the suppressive impact of PL in TDMactivated macrophages on Mincle expression and Syk phosphorylation. Our research also found that in murine alveolar macrophages, TDM could trigger NF-signalling. PL had no obvious inhibitory action on the phosphorylation of I $\kappa \beta$, unlike dexamethasone.

Our findings showed that the Mincle-Syk-ERK signalling system in TDM-stimulated alveolar macrophages was efficiently suppressed by PL. The in vivo studies involving the mouse model of granulomatous inflammation caused by TDM revealed that PL administration increased this inflammation. The pepper extract inhibited the development of pro-inflammatory cytokines, the formation of pulmonary granuloma and the inflammation caused by TDM. After 7 days of therapy with a higher dose ( $150 \mathrm{mg} / \mathrm{kg}$ b.w.), suppression was quite successful, with the amount and volume of pulmonary granulomas steadily rising and being progressively resolved (42). Consequently, TDM-treated mice can constitute an effective in vivo model for the investigation of granuloma-linked inflammation.

Our findings also revealed that the IL-10 related anti-inflammatory effect was not substantially impaired by TDM activation and treatment with PL. We found a decline in the recruitment of immune cells to reduce the level of lung damage. In agreement with an earlier report (46), macrophages were the main component of the invading cells forming granulomas, and neutrophils were present in substantially increased numbers following TDM stimulation. However, PL treatment reduced infiltrating neutrophils and macrophages, but not eosinophils. Piperlongumine demonstrated more efficient repression of invasion by neutrophils, suggesting a unique function for it against granulomatous inflammation. Our analysis, therefore, indicated that PL mitigated this inflammation caused by TDM in mice without showing harmful actions. The results indicate significant antiinflammatory activity of PL in tuberculosis.

In summary, using a TDM-triggered murine alveolar inflammation model, the study showed that PL effectively suppressed in vitro inflammatory macrophage responses by inhibiting the Mincle-Syk-ERK signalling pathway.
Furthermore, therapeutic effects of PL on pulmonary granulomatous inflammation in mice were also observed, implying its utility as adjuvant therapy complementary to antibiotics in the treatment of TB patients.

Conflict of Interests Statement: The authors declare that there is no conflict of interests regarding the publication of this article.

Financial Disclosure Statement: This study was conducted under the Project of Yunnan Science and Technology Department (Nos 2018FH001-077 and 201901N070046), Key Project of Kunming Science and Technology Bureau (No. 2019-1-N-25318000003253).

Animal Rights Statement: Before the start of the study protocol, the Institutional Animal Ethics Committee authorised all experimental protocols involving animal experimentation and acknowledged them as complying with internationally acknowledged ethical norms for the conduct of animal testing.

\section{References}

1. Abreu A.C., Coqueiro A., Sultan A.R., Lemmens N., Kim H.K., Verpoorte R., van Wamel W.J.B., Simões M., Choi Y.H.: Looking to nature for a new concept in antimicrobial treatments: isoflavonoids from Cytisus striatus as antibiotic adjuvants against MRSA. Sci Rep 2017, 7, 3777, doi: 10.1038/s41598-017-03716-7.

2. Adesina S.K., Adebayo A.S., Adesina S.K., Gröning R.: New constituents of Piper guineense fruit and leaf. Pharmazie 2003, 58, 423-425, doi: 10.1002/chin.200340213.

3. Alviano D.S., Alviano C.S.: Plant extracts: search for new alternatives to treat microbial diseases. Curr Pharm Biotechnol 2009, 10, 106-121, doi: 10.2174/138920109787048607.

4. Bilaçeroğlu S., Perim K., Büyükşirin M., Çelikten E.: Prednisolone: a beneficial and safe adjunct to antituberculosis treatment? A randomized controlled trial. Int J Tuberc Lung Dis 1999, 3, 47-54, PMID: 10094169

5. Bloch H., Sorkin E., Erlenmeyer H.: A toxic lipid component of the tubercle bacillus (cord factor). I. Isolation from petroleum ether extracts of young bacterial cultures. Am Rev Tuberc 1953, 67, 629-643, doi: 10.1164/art.1953.67.5.629.

6. Bourigault M.L., Segueni N., Rose S., Court N., Vacher R., Vasseur V., Erard F. Bert M.L., Gracia I., Iwakura Y., Jacobs M., Ryffel B., Quesniaux V.F.J.: Relative contribution of IL-1 $\alpha$, IL-1 $\beta$ and TNF to the host response to Mycobacterium tuberculosis and attenuated M. bovis B.C.G. Immun Inflamm Dis 2013, 1, 47-62, doi: 10.1002/iid3.9.

7. Chavarria D., Silva T., Magalhães e Silva D., Remião F., Borges F.: Lessons from black pepper: piperine and derivatives thereof. Expert Opin Ther Pat 2016, 2, 245-264, doi: 10.1517/13543776.2016.1118057.

8. Chittasupho C., Manikwar P., Krise J.P., Siahaan T.J., Berkland C.: cIBR effectively targets nanoparticles to LFA-1 on acute lymphoblastic T cells. Mol Pharm 2010, 7, 146-155, doi: $10.1021 / \mathrm{mp} 900185 \mathrm{u}$.

9. Chuquimia O.D., Petursdottir D.H., Periolo N., Fernández C.: Alveolar epithelial cells are critical in protection of the respiratory tract by secretion of factors able to modulate the activity of pulmonary macrophages and directly control bacterial growth. Infect Immun 2013, 81, 381-389, doi: 10.1128/IAI.00950-12.

10. Farberman M.M., Hoffmann J.W., Ryerse J.S., Demello D.E.: Diffusible signal to murine alveolar macrophages from lipopolysaccharide- and Escherichia coli-stimulated lung Type II epithelial cells. Inflamm Res 2004, 53, 475-483, doi: 10.1007/s00011-004-1289-6.

11. Fenton M.J.: Macrophages and tuberculosis. Curr Opin Hematol 1998, 5, 72-78, doi: 10.1097/00062752-199801000-00012. 
12. Flynn J.L., Chan J., Lin P.L.: Macrophages and control of granulomatous inflammation in tuberculosis. Mucosal Immunol 2011, 4, 271-278, doi: 10.1038/mi.2011.14.

13. Guidry T.V., Olsen M., Kil K.S., Hunter R.L. Jr., Geng Y.J., Actor J.K.: Failure of CD1D-/- mice to elicit hypersensitive granulomas to mycobacterial cord factor trehalose 6,6'dimycolate. J Interferon Cytokine Res 2004, 24, 362-371, doi: 10.1089/107999004323142222.

14. Hasan A., Sadoh D., Palmer R., Foo M., Marber M., Lehner T.: The immune responses to human and microbial heat shock proteins in periodontal disease with and without coronary heart disease. Clin Exp Immunol 2005, 142, 585-594, doi: 10.1111/j.1365-2249.2005.02953.x.

15. Hemaiswarya S., Kruthiventi A.K., Doble M.: Synergism between natural products and antibiotics against infectious diseases. Phytomedicine 2008, 15, 639-652, doi: 10.1016/j.phymed. 2008.06.008.

16. Hunter R.L., Olsen M.R., Jagannath C., Actor J.K.: Multiple roles of cord factor in the pathogenesis of primary, secondary, and cavitary tuberculosis, including a revised description of the pathology of secondary disease. Ann Clin Lab Sci 2006, 36, 371-386, PMID: 17127724.

17. Kaneda K., Sumi Y., Kurano F., Kato Y., Yano I.: Granuloma formation and hemopoiesis induced by C36-48-mycolic acidcontaining glycolipids from Nocardia rubra. Infect Immun 1986, 54, 869-875, doi: 10.1128/iai.54.3.869-875.1986.

18. Karsha P.V., Lakshmi O.B.: Antibacterial activity of black pepper (Piper nigrum Linn.) with special reference to its mode of action on bacteria. Indian J Nat Prod Resour 2010, doi: 10.1128/IAI.68.5.2976-2978.2000.

19. Kurashima K., Mukaida N., Fujimura M., Yasui M., Nakazumi Y., Matsuda T., Matsushima K.: Elevated chemokine levels in bronchoalveolar lavage fluid of tuberculosis patients. Am J Respir Crit Care Med 1997, 155, 1474-1477, doi: 10.1164/ajrccm.155.4.9105097.

20. Lambert R.J.: Susceptibility testing: inoculum size dependency of inhibition using the Colworth MIC technique. J Appl Microbiol 2000, 89, 275-279, doi: 10.1046/j.1365-2672.2000.01105.x.

21. Lee W.-B., Kang J.-S., Yan J.-J., Lee M.S., Jeon B.-Y., Cho S.-N., Kim Y.-J.: Neutrophils Promote Mycobacterial Trehalose Dimycolate-Induced Lung Inflammation via the Mincle Pathway. PLoS Pathog 2012, 8, e1002614, doi: 10.1371/ journal.ppat.1002614.

22. Mgbeahuruike E.E., Yrjönen T., Vuorela H., Holm Y.: Bioactive compounds from medicinal plants: Focus on Piper species. S Afr J Bot 2017, 112, 54-69, doi: 10.1016/j.sajb.2017.05.007.

23. Mirza Z.M., Kumar A., Kalia N.P., Zargar A., Khan I.A.: Piperine as an inhibitor of the MdeA efflux pump of Staphylococcus aureus. J Med Microbiol 2011, 60, 1472-1478, doi: 10.1099/jmm.0.033167-0.

24. Mohammed K.A., Nasreen N., Ward M.J., Mubarak K.K., Rodriguez-Panadero F., Antony V.B.: Mycobacterium-mediated chemokine expression in pleural mesothelial cells: role of C-C chemokines in tuberculous pleurisy. J Infect Dis 1998, 178, 1450-1456, doi: 10.1086/314442.

25. Naika R., Prasanna K., Ganapathy P.J.P.: Antibacterial activity of piperlongumine an alkaloid isolated from methanolic root extract of Piper longum L. Pharmacophore 2010, 1, 141-148.

26. Orme I.M., Robinson R.T., Cooper A.M.: The balance between protective and pathogenic immune responses in the TB-infected lung. Nat Immunol 2015, 16, 57-63, doi: 10.1038/ni.3048.

27. Hernando-Amado S., Alcalde-Rico M., Gil-Gil T., Valverde J.R., Martínez J.L.: Naringenin Inhibition of the Pseudomonas aeruginosa Quorum Sensing Response Is Based on Its TimeDependent Competition With N-(3-Oxo-dodecanoyl)-Lhomoserine Lactone for LasR Binding. Front Mol Biosci 2020, 7 , 25, doi: 10.3389/fmolb.2020.00025.

28. Ozeki Y., Tsutsui H., Kawada N., Suzuki H., Kataoka M., Kodama T., Yano I., Kaneka K., Kobayashi K.: Macrophage scavenger receptor down-regulates mycobacterial cord factorinduced proinflammatory cytokine production by alveolar and hepatic macrophages. Microb Pathog 2006, 40, 171-176, doi: 10.1016/j.micpath.2005.12.006.
29. Philipova I., Valcheva V., Mihaylova R., Mateeva M., Doytchinova I., Stavrakov G.: Synthetic piperine amide analogs with antimycobacterial activity. Chem Biol Drug Des 2018, 91, 763-768, doi: 10.1111/cbdd.13140.

30. Piska K., Gunia-Krzyżak A., Koczurkiewicz P., Wójcik-Pszczoła K., Pękala E.: Piperlongumine (piplartine) as a lead compound for anticancer agents - Synthesis and properties of analogues: A mini-review. Eur J Med Chem 2018, 156, 13-20, doi: 10.1016/j.ejmech.2018.06.057.

31. Ramakrishnan L.: Revisiting the role of the granuloma in tuberculosis. Nat Rev Immunol 2012, 12, 352-366, doi: 10.1038/nri3211.

32. Sadek M.I., Sada E., Toossi Z., Schwander S.K., Rich E.A.: Chemokines induced by infection of mononuclear phagocytes with mycobacteria and present in lung alveoli during active pulmonary tuberculosis. Am J Respir Cell Mol Biol 1998, 19, 513-521, doi: 10.1165/ajrcmb.19.3.2815.

33. Sakamoto K.: The pathology of Mycobacterium tuberculosis infection. Vet Pathol 2012, 49, 423-439, doi: 10.1177/ 0300985811429313.

34. Sasindran S.J., Torrelles J.B.: Mycobacterium Tuberculosis Infection and Inflammation: what is Beneficial for the Host and for the Bacterium? Front Microbiol 2011, 2, 2, doi: 10.3389/ fmicb.2011.00002.

35. Schoenen H., Bodendorfer B., Hitchens K., Manzanero S., Werninghaus K., Nimmerjahn F., Agger E.M., Stenger S., Andersen P., Ruland J., Brown G.D., Wells C.H., Lang R.: Cutting edge: Mincle is essential for recognition and adjuvanticity of the mycobacterial cord factor and its synthetic analog trehalosedibehenate. J Immunol 2010, 184, 2756-2760, doi: 10.4049/jimmunol.0904013.

36. Stavri M., Piddock L.J., Gibbons S.: Bacterial efflux pump inhibitors from natural sources. J Antimicrob Chemother 2007, 59, 1247-1260, doi: 10.1093/jac/dk1460.

37. Subramani R., Narayanasamy M., Feussner K.D.: Plant-derived antimicrobials to fight against multi-drug-resistant human pathogens. 3 Biotech 2017, 7, 172, doi: 10.1007/s13205-0170848-9.

38. Sunil V.R., Patel K.J., Nilsen-Hamilton M., Heck D.E., Laskin J.D., Laskin D.L.: Acute endotoxemia is associated with upregulation of lipocalin $24 \mathrm{p} 3 / \mathrm{Lcn} 2$ in lung and liver. Exp Mol Pathol 2007, 83, 177-187, doi: 10.1016/j.yexmp.2007.03.004.

39. Tao F., Kobzik L.: Lung macrophage-epithelial cell interactions amplify particle-mediated cytokine release. Am J Respir Cell Mol Biol 2002, 26, 499-505, doi: 10.1165/ajrcmb.26.4.4749.

40. Tegos G., Stermitz F.R., Lomovskaya O., Lewis K.: Multidrug pump inhibitors uncover remarkable activity of plant antimicrobials. Antimicrob Agents Chemother 2002, 46, 3133-3141, doi: 10.1128/AAC.46.10.3133-3141.2002.

41. Tsao T.C., Hong J., Huang C., Yang P., Liao S.K., Chang K.S.: Increased TNF-alpha, IL-1 beta and IL-6 levels in the bronchoalveolar lavage fluid with the upregulation of their mRNA in macrophages lavaged from patients with active pulmonary tuberculosis. Tuber Lung Dis 1999, 79, 279-285, doi: 10.1054/tuld.1999.0215.

42. Van Vuuren S.F., Suliman S., Viljoen A.M.: The antimicrobial activity of four commercial essential oils in combination with conventional antimicrobials. Lett Appl Microbiol 2009, 48, 440-446, doi: 10.1111/j.1472-765X.2008.02548.x.

43. Wei L., Wu J., Liu H., Yang H., Rong M., Li D., Zhang P., Han J., Lai R.: A mycobacteriophage-derived trehalose-6,6'dimycolate-binding peptide containing both antimycobacterial and anti-inflammatory abilities. FASEB J 2013, 27, 3067-3077, doi: 10.1096/fj.13-227454.

44. Yang S.-K., Low L.-Y., Yap P.S.-X., Yusoff K., Mai C.-W., Lai K.-S.: Plant-Derived Antimicrobials: Insights into Mitigation of Antimicrobial Resistance. Rec Nat Prod 2018, 12, 295-316, doi: 10.25135/rnp.41.17.09.058.

45. Yi Y.-S., Son Y.-J., Ryou C., Sung G.-H., Kim J.-H., Cho J.Y.: Functional roles of Syk in macrophage-mediated inflammatory responses. Mediators Inflamm 2014, 270302, doi: $10.1155 / 2014 / 270302$. 